\title{
In eigener Sache: BHM - Neuerungen im Redaktionsteam
}

Online publiziert am 6. Juni 2014

Sehr geehrter Leserinnen und Leser!

Die Berg- und Hüttenmännischen Monatshefte sind die einzige wissenschaftliche österreichische Zeitschrift für die Fachbereiche Bergwesen, Metallurgie und Werkstofftechnik. In den letzten Jahren ist es gelungen, die Anzahl der Publikationen in den Heften zu steigern. Es ist für uns eine stetige Herausforderung, die Qualität und Informationsinhalte sowohl im wissenschaftlichen wie auch im technischen Bereich zu verbessern.

In den letzten beiden Jahren wurden einzelne Hefte thematischen Schwerpunkten in Form von Sonderausgaben zu Tagungen, Konferenzen oder Symposien gewidmet, sowie Fachhefte von Gastredakteuren (Guest Editors) zu fachlichen Schwerpunktthemen gestaltet. Die Rückmeldung der Leser dazu war sehr positiv, und wir wollen daher diese Strategie in Zukunft verstärkt fortführen. Erfreulich ist auch die Entwicklung der internationalen Verbreitung der BHM durch den Online-Auftritt. Im Jahr 2012 betrug die Anzahl der Volltext-Downloads von Beiträgen 14.226, wobei fast $50 \%$ aus nicht deutschsprachigen Ländern und davon wieder $25 \%$ aus dem Raum Asien-Pazifik und $11 \%$ aus Nordamerika kamen.

Um diese positive Entwicklung verstärkt fortführen zu können, haben wir das Redaktionsteam der BHM mit Anfang 2014 neu organisiert. Die Leitung desTeams haben zwei Hauptredakteure (Editors-in-Chief) inne. Insgesamt
15 Fachredakteure (Section Editors), die Expertise in den Kernfachbereichen Bergwesen, Metallurgie und Werkstofftechnik, aber auch wichtigen angrenzenden Fachbereichen repräsentieren, sorgen für eine stärkere fachliche Ausrichtung. Die Fachredakteure werden in Zukunft stärker bei der Gestaltung der BHM eingebunden sein, so z. B. als Gastredakteur von Fachheften und Sonderausgaben. Unterstützt werden sie von den Mitgliedern des Beirates (Advisory Board), wobei jeder Fachbereich durch zwei bis drei Personen im Beirat vertreten wird. Bei der Auswahl der Fachredakteure und Beiratsmitglieder wurden die Aspekte der internationalen Ausrichtung und der genderspezifischen Ausgewogenheit so weit wie möglich berücksichtigt.

Wir hoffen, dass wir durch die Neuorganisation des Redaktionsteams die Qualität der BHM weiter steigern und Sie als interessierte Leserinnen und Leser damit noch besser erreichen können.

\section{Glück auf!}

\section{Gerhard Mayer}

Johannes Schenk 